Journal of Applied Finance \& Banking, Vol. 11, No. 2, 2021, 109-127

ISSN: 1792-6580 (print version), 1792-6599(online)

https://doi.org/10.47260/jafb/1124

Scientific Press International Limited

\title{
Covid-19 and the Technology Bubble 2.0: Evidence from DCC-MGARCH and Wavelet Approaches
}

\author{
Caner Özdurak ${ }^{1}$ and Cengiz Karataş²
}

\begin{abstract}
There has probably never been as big a divergence between markets and economies as there is in the pandemic period. This paper is an attempt to test the 'time-varying' and 'time-scale dependent' volatilities of major technology stocks, FAANG and Microsoft, for analyzing the possibility of a second technology bubble in the markets. Consistent with the results of DCC-GARCH models, our analysis based on the application of the Wavelet approach also indicates that major technology behave and move as if they were all one stock in the pandemic period which makes us to be cautious about a second dotcom crisis since \%26 of S\&P 500 market cap is driven by FAANG and Microsoft stocks.
\end{abstract}

JEL classification numbers: C58, D53, O14.

Keywords: Dot-com crisis, tech bubble, DCC-GARCH, FAANG, Wavelet.

\footnotetext{
1 Assistant Professor. Yeditepe University, Department of Financial Economics, Ataşehir, Istanbul, Turkey. ORCID: 0000-0003-0793-7480.

${ }^{2}$ Research Assistant. Yeditepe University, Department of Financial Economics, Ataşehir, Istanbul, Turkey. ORCID: 0000-0001-7554-801X.
}

Article Info: Received: December 14, 2020. Revised: December 30, 2020.

Published online: January 4, 2021. 


\section{Introduction}

A significant number of researchers focused on the impact of Covid-19 to financial markets. Mazur et al. (2020) investigate the US stock market performances during the crash of March 2020. Mirza et al. (2020) assess the price reaction, performance, and volatility timing of European investment funds during the outbreak of Covid19. Gong et al. (2020) mentioned that the flu pandemic (HIN1) prompted financial intermediation inefficiency with an increase in loan spreads. Goldman Sachs neologized the abbreviation FAAMG, which is Facebook, Amazon, Apple, Microsoft, and Google in 2017. A significant portion of S\&P500 Index market cap is driven by big tech companies. This high impact made us cautious about the existence of a possible bubble and a second dotcom crisis possibility. As of July 2020 S\&P 500 has six companies (FAANG-M) responsible for over 26\% (Facebook $3 \%$, Amazon 6\%, Apple 7\%, Netflix 1\%, Alphabet (Google) 4\% and Microsoft 6\%) of the index's rebound worse even than the dot com bubble ${ }^{3}$ of 1999/2000.

\section{Methodology}

Two approaches have been identified for this study. GARCH and DCC estimations are utilized to model returns and variance of commodities and cross linkages (Table4-5-6). Since all the series in our dataset are highly leptokurtic, we chose to use t-distribution in our GARCH models to capture fat-tailed issue. The use of methodologies for wavelet transformation requires no predictions and is equal to generating more practical outcomes (In and Kim, 2013). Wavelet method is used to detect co-movement between time series. It distinguishes by extending on time and frequency domain. By these properties, wavelet analysis has defined a wide variety of application fields. Quantitative performance in wavelet analysis systems, in particular, was improved by the studies of Torrence-Compo and Lau-Weg. Torrence and Compo (1998) enhanced the latest statistical significance tests of Lau and Weg (1995) by building significance thresholds and confidence intervals, as well as by defining correlation and cross-wavelet spectra in analysis focused on atmospheric time series. Concentrating on wavelet methods of time series by the tests on cross wavelet transform and extended by Grinsted et al.(2004). They illustrated the wield of phase angle statistics to check faith in random relationships by using expanded wavelet software packages for geophysical time sequences. Via cross-wavelet method, Tiwari (2012) studied the relation between share prices and interest rates in the Indian economy and industrial production, oil prices and inflation in the German economy. Barunik et al. (2012), (2013) discussed energy commodities comovements, European stock markets and exchanged assets such as oil, gold, stocks,

\footnotetext{
3 The dot-com bubble is also known as the tech bubble. This balloon was a stock market bubble created by extreme speculation in Internet-related businesses in the late 1990s.Although Dotcom Balloon was largely referred to as the internet companies' shares were destroyed in the stock market, one of the factors that actually inflated the bubble was the dreams of smart devices that did not exist at that time.
} 
examining their structures and contrasting their findings with standard econometric instruments.

\subsection{DCC-GARCH}

The Dynamic Conditional Correlation (DCC-GARCH) belongs to the class" Models of conditional variances and correlations. It was introduced by $\mathrm{f}$ and Sheppard in (2001). The idea of the models in this class is that the covariance matrix, $\mathrm{Ht}$, can be decomposed into conditional standard deviations, Dt, and a correlation matrix, Rt. In the DCC-GARCH model both Dt and Rt are designed to be timevarying.

Suppose we have returns, at, from $\mathrm{n}$ assets with expected value 0 and covariance matrix $\mathrm{H}_{\mathrm{t}}$. Then the Dynamic Conditional Correlation (DCC-) GARCH model is defined as:

$r_{t}=\mu_{t}+\alpha_{t}$

$\alpha_{t}=H_{t}^{1 / 2} z_{t}$

$H_{t}=D_{t} R_{t} D_{t}$

$\mathrm{r}_{\mathrm{t}}: \mathrm{n} \times 1$ vector of $\log$ returns of $\mathrm{n}$ assets at time $\mathrm{t}$,

$\alpha_{t}: E\left[\alpha_{t}\right]=0$ and $\operatorname{Cov}\left[\alpha_{t}\right]=H_{t} n \times 1$ vector of mean-corrected returns of $n$ assets at time t, i.e.,

$\mu_{t:} n \times 1$ vector of the expected value of the conditional $r_{t}$

$\mathrm{H}_{\mathrm{t}}: \mathrm{n} \times \mathrm{n}$ matrix of conditional variances of $\alpha_{\mathrm{t}}$ at time $\mathrm{t}$.

$\mathrm{H}_{\mathrm{t}}^{1 / 2}$ : Any $\mathrm{n} \times \mathrm{n}$ matrix at time $\mathrm{t}$ such that $\mathrm{H}_{\mathrm{t}}$ is the conditional variance matrix of at. $\mathrm{H}_{\mathrm{t}}{ }^{1 / 2}$ may be obtained by a Cholesky factorization of $\mathrm{H}_{\mathrm{t}}$.

$D_{t}: n \times n$, diagonal matrix of conditional standard deviations of $\alpha_{t}$ at time $t$

$\mathrm{R}_{\mathrm{t}}: \mathrm{n} \times \mathrm{n}$ conditional correlation matrix of $\alpha_{\mathrm{t}}$ at time $\mathrm{t}$

$Z_{\mathrm{t}}: \mathrm{n} \times 1$ vector of iid errors such that $\mathrm{E}\left[\mathrm{Z}_{\mathrm{t}}\right]=0$ and $\mathrm{E}\left[\mathrm{Z}_{\mathrm{t}}^{\mathrm{T}}\right]$

In addition, $\mathrm{Q}_{0}$, the starting value of $\mathrm{Q}_{\mathrm{t}}$, has to be positive definite to guarantee $\mathrm{H}_{\mathrm{t}}$ to be positive definite. The correlation structure can be extended to the general DCC $(\mathrm{M}, \mathrm{N})-\mathrm{GARCH}$ model:

$$
\begin{aligned}
& R_{t}=\varrho_{t}^{* 1} \varrho_{t} \varrho_{t}^{* 1} \\
& \varrho_{t}=\left(1-\varrho_{1}-\varrho_{2}\right) \bar{\varrho}+\varrho_{1} \varepsilon_{t-1} \varepsilon_{t-1}^{T}+\varrho_{2} \varrho_{t-1}
\end{aligned}
$$

In this context $\varrho_{t}$ can be estimated as mentioned below:

$$
\varrho_{t}=\frac{1}{T} \sum_{t=1}^{T} \varepsilon_{t} \varepsilon_{t}^{T}
$$


There are imposed some conditions on the parameters $\varrho_{1}$ and $\varrho_{2}$ to guarantee $\mathrm{H}_{\mathrm{t}}$ to be positive definite. In addition to the conditions for the univariate GARCH model to ensure positive unconditional variances, the scalars $a$ and $b$ must satisfy: $\varrho_{1} \geq 0, \varrho_{2} \geq 0$ ve $\varrho_{1}+\varrho_{2}<1$

\subsection{Wavelet Analysis}

In this research, Wavelet coherence was used to understand correlations of big tech companies. We measure the series co-movements via wavelet coherence. Coherence areas represented by red to blue colors are seen in wavelet coherence figures, which display high-level to low-level correlation between two series on given period. Phase angle offer additional detail on causal relationships. In figures, we have arrows to look at the co-movement of the series. If the arrows move right for a time interval, so they are in phase, they co-move in that time interval. We applied the wavelet package invented by Grinsted et al. (2004) for two time series in our analysis. The time series of the CWT (Continuous Wavelet Transform) can be completely decomposed and then reconstructed. CWT is especially useful for the purpose of extraction of features.

CWT works as a band pass sieve for data set $x(t)$ and be described with $W_{x}(\tau, s)=\frac{1}{\sqrt{s}} \sum_{t=1}^{N} x(t) \varphi^{*}\left(\frac{t-\tau}{s}\right)$,

where $*$ is complex conjugate.

The Morlet Wavelets are also used and specified which was advertised in Morlet Goupillaud, Grossman [1984] as;

$\varphi(\eta)=\pi^{-1 / 4} e^{i \omega_{0} \eta} e^{-1 / 2 \eta^{2}}$,

$\eta$ is time and $\omega_{0}$ is frequency and selected 6 because it offer better balance among frequency localization and time (Grinsted et al.,2004). For many factors, such as scale to frequency transformation facility, numerical advantages, low are Heisenberg box and excellent balance among frequency and time, Morlet Wavelets are particularly preferred.

\subsubsection{Wavelet Coherence (WTC)}

Cross-Wavelet Transform is constituted by CWT's and reveals mutual power and consistent phase in frequency-time space to these series.

Cross-wavelet transform is described; $\Psi_{n}^{X Y}(s)=W_{n}^{X}(s) . W_{n}^{Y *}(\mathrm{~s})$. 
Where, $W_{n}^{X}(s)$ and $W_{n}^{Y}(\mathrm{~s})$, are CWTs of X and Y. $W_{n}^{Y *}(\mathrm{~s})$; complex conjugate of $W_{n}^{Y}(\mathrm{~s})$. So WTC be defined as;

$$
R_{n}^{2}(s)=\frac{\left|S\left(s^{-1} \Psi_{n}^{X Y}(s)\right)\right|^{2}}{s\left(s^{-1}\left|W_{n}^{X}(s)\right|^{2}\right) \cdot S\left(s^{-1} \mid\left(\left.W_{n}^{Y}(s)\right|^{2}\right)\right.}
$$

Here, $\mathrm{s}$ is a wavelet scale, $\mathrm{S}$ is smoothing operator,

\subsubsection{Phase}

Wavelet coherence phase difference is;

$$
\phi_{x y}(k, \mathrm{~s})=\tan ^{-1}\left(\frac{I\left\{S\left(\left(s^{-1} \Psi_{n}^{X Y}(k, s)\right)\right)\right\}}{R\left\{S\left(\left(s^{-1} \Psi_{n}^{X Y}(k, s)\right)\right)\right\}}\right), \phi_{x y} \in[-\pi, \pi],
$$

$\mathrm{R}$ and I are real and imaginary parts. The co-movement of two series at different scales can be seen by phase differences. If they have co-moved and in phase the arrows point right.

\section{Data and Empirical Findings}

Our dataset contains daily Facebook (FACEBOOK) Alphabet-Google (ALPHABET), Apple (APPLE), Amazon (AMAZON), Netflix (NETFLIX) and Microsoft (MICROSOFT) between June 24, 2016, and July 6, 2020, for FAANG and FAAMG analysis. We also narrowed the period between December 31, 2019, and July 6, 2020, to analyze the impact of Covid-19 on technology stock performance. We will construct our FAANG and FAAMG GARCH models separately for both periods based on this dataset. In Figure 1, normalized daily stock prices of Facebook, Apple, Amazon, Alphabet, Netflix, and Microsoft are exhibited. In the Covid-19 period, we can see the FAANG stocks and Microsoft stocks behave and move as if they are all one stock. Their movement is very identical. There has probably never been as big a divergence between markets and economies as there is in the pandemic period. Even the International Monetary Fund (IMF) warned of a worrying "disconnect" between markets and the real economy. The feature of the current market dynamic is passive investing, whereby investors or funds buy indices rather than individual stocks. This may be distorting the current cycle. The FAANG stocks are seemingly sucking in a level of investment that bears little relation to their inherent value or projected future earnings. 
Overall Period

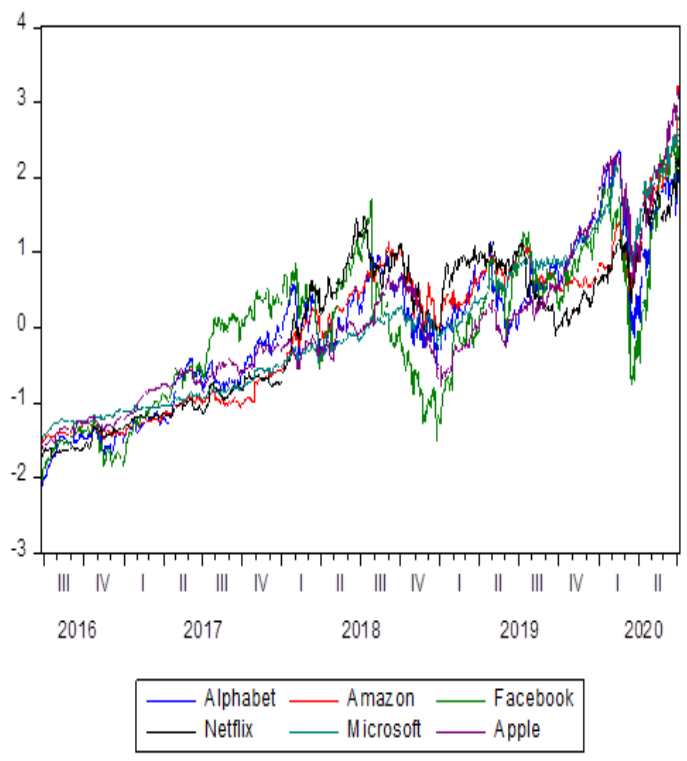

Covid-19 Period

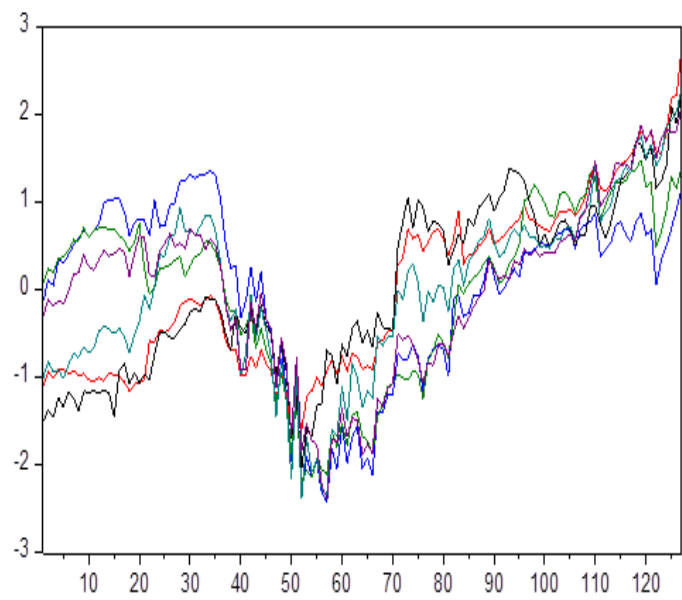

Figure 1: FAANG and Microsoft Normalized Daily Stock Prices

In Table $1 \varrho_{1}$ and $\varrho_{2}$ dynamic conditional correlation coefficients are exhibited for all GARCH models in every period. A DCC model really should only be applied to a set of series which are relatively similar since the cross-correlations are all governed by just two parameters. If $Q_{2}$ is very close to 1 , then the process is closer to being a CC. The "dynamic" part comes from $\varrho_{1}$. However, in practice, a "large" value for DCC $\varrho_{1}$ is something like .1 to .2, with @2 being relatively close to 1-@2. If both $\varrho_{1}$ and $\varrho_{2}$ are small, it means that there appears to be no systematic correlation among the variables. According to Francq and Zakoian (2010), there are two definitions regarding the GARCH process. The first one is called semi-strong, where there exists the coefficient of the constant, arch and GARCH (no need to be positive, but must be significant). The second one is called a strong GARCH process, where the coefficient of arch and GARCH are nonnegative while the coefficient of the constant must be positive. According to FAANG models for the overall period, Facebook-Netflix GARCH process is semi-strong while AmazonNetflix GARCH process is strong and $\varrho_{1}$ is 0.1160 and $\varrho_{2}$ is 0.6253 . Apple-Netflix and Alphabet-Netflix GARCH processes are not significant. For the pandemic period FAANG models, Facebook-Netflix GARCH process is still semi-strong as well as Alphabet -Netflix model. But in Alphabet-Netflix model @2 is 0.9967 which shows that the process is not dynamic but a CC process. Moreover, Apple-Netflix and Amazon-Netflix GARCH processes are not significant in the Covid-19 period. 
Table 1: FAANG DCC GARCH Model Results

FAANG Models-DCC GARCH Tables (Overall Period)

\begin{tabular}{|c|c|c|c|c|}
\hline Facebook-Netflix & Coefficients & Z-statistics & Probability & AIC \\
\hline$\varrho_{1}$ & 0.00 & -24097.8600 & 0.00 & 6.03 \\
\hline$\varrho_{2}$ & 0.59 & 7.7565 & 0.00 & \\
\hline Observations & 997 & & & \\
\hline Apple-Netflix & Coefficients & Z-statistics & Probability & AIC \\
\hline$\varrho_{1}$ & 0.03 & 1.0458 & 0.30 & 5.77 \\
\hline$\varrho_{2}$ & 0.65 & 1.5774 & 0.11 & \\
\hline Observations & 998 & & & \\
\hline Alphabet-Netflix & Coefficients & Z-statistics & Probability & AIC \\
\hline$\varrho_{1}$ & -0.01 & -3659.6670 & 0.00 & 5.87 \\
\hline$\varrho_{2}$ & 0.58 & 0.4693 & 0.64 & \\
\hline \multicolumn{5}{|l|}{ Observations } \\
\hline Amazon-Netflix & Coefficients & Z-statistics & Probability & AIC \\
\hline$\varrho_{1}$ & 0.12 & 3.67 & 0.00 & 5.70 \\
\hline$\varrho_{2}$ & 0.62 & 5.87 & 0.00 & \\
\hline Observations & 998 & & & \\
\hline
\end{tabular}

FAANG Models-DCC GARCH Tables (Covid-19 Period)

Facebook-Netflix Coefficients Z-statistics Probability AIC

$\begin{array}{lcccc}\varrho_{1} & -0.01 & -199.6 & 0.00 & 5.57 \\ \varrho_{2} & 0.77 & 4.2 & 0.00 & \end{array}$

Observations $\quad 127$

Apple-Netflix Coefficients Z-statistics Probability AIC

$\begin{array}{lllll}Q_{1} & 0.02 & 0.34 & 0.74 & 5.73 \\ Q_{2} & 0.82 & 1.45 & 0.15 & \end{array}$

Observations $\quad 127$

Alphabet-Netflix Coefficients Z-statistics Probability AIC $\begin{array}{lllll}\varrho_{1} & -0.06 & -2.05 & 0.04 & 5.68\end{array}$

$Q_{2} \quad \begin{array}{llll}1.00 & 37.36 & 0.00\end{array}$

Observations $\quad 127$

Amazon-Netflix Coefficients Z-statistics Probability AIC

$\begin{array}{ccccc}\varrho_{1} & 0.10 & 0.83 & 0.40 & 5.56 \\ \varrho_{2} & 0.33 & 0.58 & 0.56 & \\ \text { Observations } & 127 & & & \end{array}$

In Table 2 based on FAAMG models, we can claim that Apple-Microsoft and Alphabet-Microsoft GARCH processes are statistically significant. $Q_{1}$ is 0.0265 and $\varrho_{2}$ is 0.9651 for Appel-Microsoft and $\varrho_{1}$ is 0.0109 and $\varrho_{2}$ is 0.9666 in Alphabet which refers to CC processes, not dynamic. Furthermore, only @2 is significant in the Facebook-Microsoft and $\mathrm{Q}_{1}$ is significant in the Amazon-Microsoft model.

In this context movement of conditional correlation of FAANG and FAAMG stock returns are depicted in Figure 2. For FAANG models, graphs show that in the overall period correlations between Apple-Netflix and Amazon-Netflix are highly volatile and vary substantially over time. The correlation goes through several troughs and peaks and a reverse sign recurs. For Facebook-Netflix and AlphabetNetflix we see one-time spikes and in the overall period, the volatility is low for mentioned pairs. Time-varying conditional correlations exhibit a relatively higher level of co-movement between Facebook-Netflix and Alphabet-Netflix pairs. For FAAMG models, graphs show that in the overall period correlations between Amazon-Microsoft are highly volatile and vary substantially over time. The correlation goes through several troughs and peaks and a reverse sign recurs. Timevarying conditional correlations exhibit a relatively higher level of co-movement between Facebook-Microsoft. 
Table 2: FAAMG DCC GARCH Model Results

FAAMG Models-DCC GARCH Tables (Overall Period)

\begin{tabular}{lrrrr}
\hline Facebook-Microsoft & Coefficients & Z-statistics & Probability & AIC \\
$\mathrm{Q}_{1}$ & -0.01 & -1.28 & 0.20 & 5.84 \\
$\mathrm{Q}_{2}$ & 0.59 & 3.31 & 0.00 & \\
Observations & 998 & & &
\end{tabular}

Apple-Microsoft

$Q_{1}$

$Q_{2}$

Observations

Amazon-Microsoft

$Q_{1}$

$\mathrm{Q}_{2}$

Observations

Alphabet-Microsoft

$Q_{1}$

$\mathrm{Q}_{2}$

Observations

$\begin{array}{rrrr}\text { Coefficients } & \text { Z-statistics } & \text { Probability } & \text { AIC } \\ 0.03 & 3.75 & 0.00 & 5.57 \\ 0.97 & 80.48 & 0.00 & \end{array}$

998

Wavelet coherence plots in Figure 3, tells that, coherence areas (red regions) exist in various scales especially at medium run. The most coherent areas almost at 8128 days till Apr.2017(day 200) and around Feb.2020(day 900) which is the COVID-19 Period. In all figures, the series shows higher correlations around Feb.2020(day 900), the arrows point right means, all series co-moved in that periods. The most correlated area is on WTC: Amazon-Microsoft (red areas) means the correlation between them is very high with respect to other series in overall period. Another higher correlated time series by the wavelet coherence figures are Google-Microsoft, Amazon-Netflix, Facebook-Netflix and Netflix-Google. In COVID period all series highly correlated since the red regions after the day number 900, we see that same results for all pairs. If we look at the plots in Figure 4 for the wavelet coherence results of technology stocks for understanding their behavior easily on that period, all pairs have extremely high correlation areas on long run for whole COVID-19 period, and the arrows points right means they co-move in this time interval. The higher co-movement results in order on Apple-Microsoft, Facebook-Google, Amazon-Microsoft, Google-Microsoft, Amazon-Netflix and Apple-Google. Above all, Apple-Microsoft pairs co-move excessively on COVID19 period (If we look at Apple-Microsoft pair, the whole graphic is red). 


\section{FAANG-Overall Period Conditional Correlations}

Facebook-Netflix

RHO12
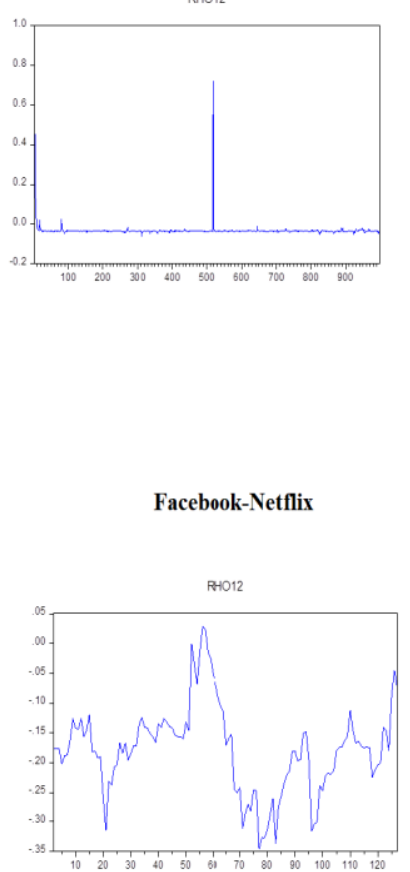

Apple-Netflix

RHO12

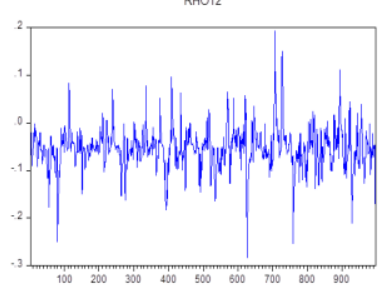

Amazon-Netflix

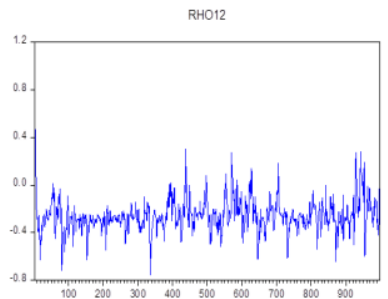

Alphabet (Google)-Netflix

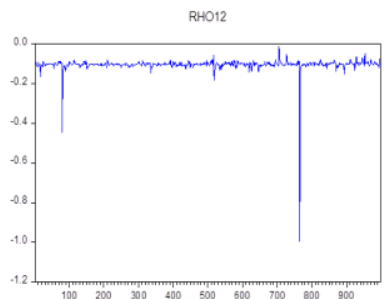

FAANG-Covid 19 Period Conditional Correlations

Apple-Netflix

RHO12

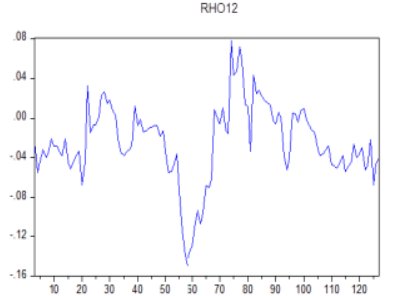

Amazon-Netflix

RHO12

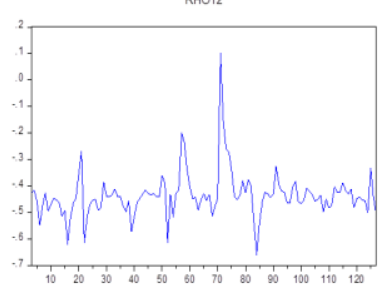

Alphabet (Google)-Netflix

RHO12

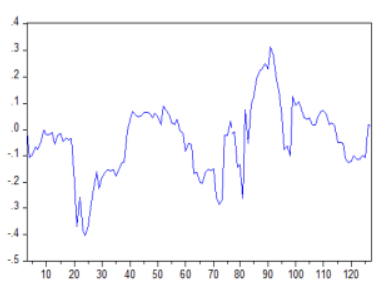

FAAMG-Overall Period Conditional Correlations

Facebook-Microsoft

RHO12

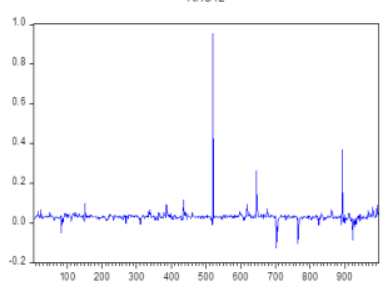

Apple-Microsoft

RHO12

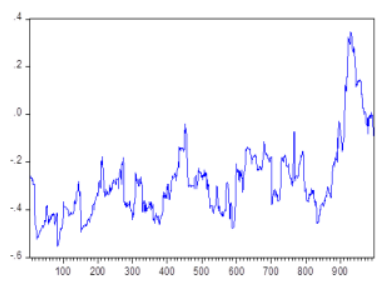

Amazon-Microsoft

RHO12

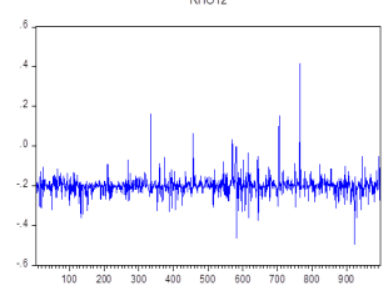

Alphabet (Google)-Microsoft

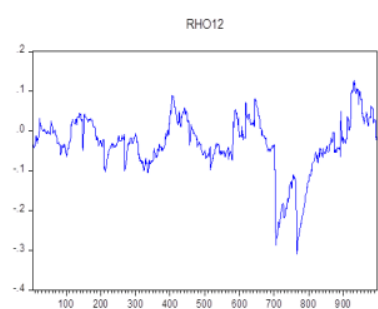

Figure 2: Conditional Correlation Graphs 


\section{Conclusion}

There has probably never been as big a divergence between markets and economies as there is in the pandemic period. At its peak in April 2020, the pandemic forced an estimated 3 billion people worldwide to be in lockdown. This situation made businesses and consumers have little choice but to use online services. Consequently, stocks of Apple, Netflix, Microsoft, and Amazon all trading at, or near record highs in the Covid-19 pandemic period. Investors believe the business models of these companies can not only weather this downturn but also thrive in it, so they rushed into these stocks. Microsoft, Netflix, and Amazon have subscriptionbased services driving recurring revenue thus, a strong balance sheet and recurring revenue are what makes these companies attractive. Associated with WTC Figures, who wants to take portfolio of FAANGM, he or she not hold that investment for 8128 days around year 2017 and in long run for Covid-19 period. Especially, the Apple-Microsoft should not be hold in the same time for COVID period for portfolio diversification because they have the highest co-movement. In conclusion, WTC figures shows that series have very high coherence areas on COVID-19 period, so the Wavelet Coherence outcomes are also coherent with DCC-GARCH results and they refer, FAANGM prices co-moved in COVID-19 period. 

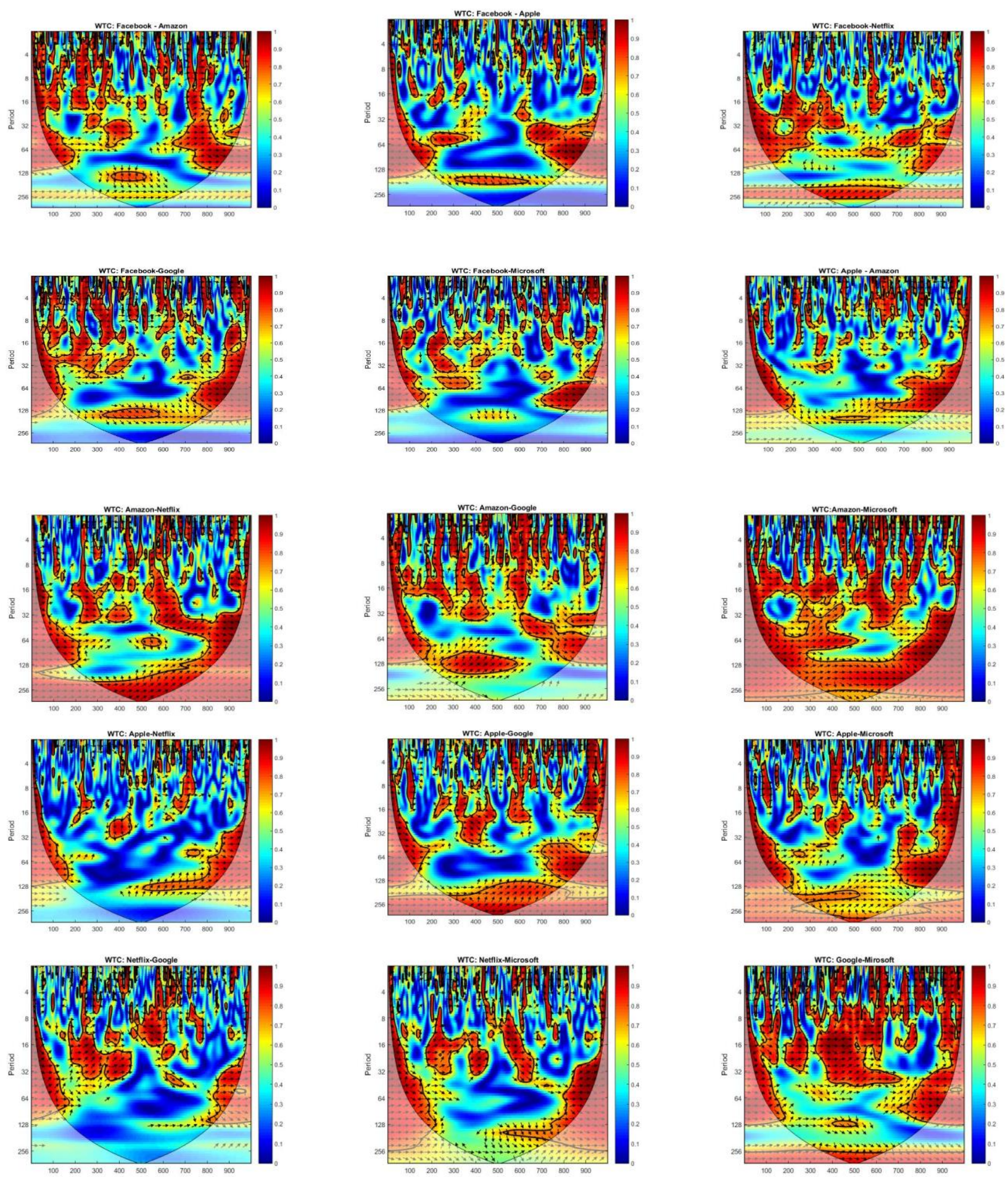

Figure 3: WTC: Facebook-Amazon-Apple-Netflix-Google-Microsoft. Date for horizontal axis are 100: Nov.2016, 200:Apr.2017, 300:Sep.2017, 400:Feb.2018, 500:Jun.2018, 600:Nov.2018, 700:Apr.2019, 800:Sep.2019, 900:Feb.2020 

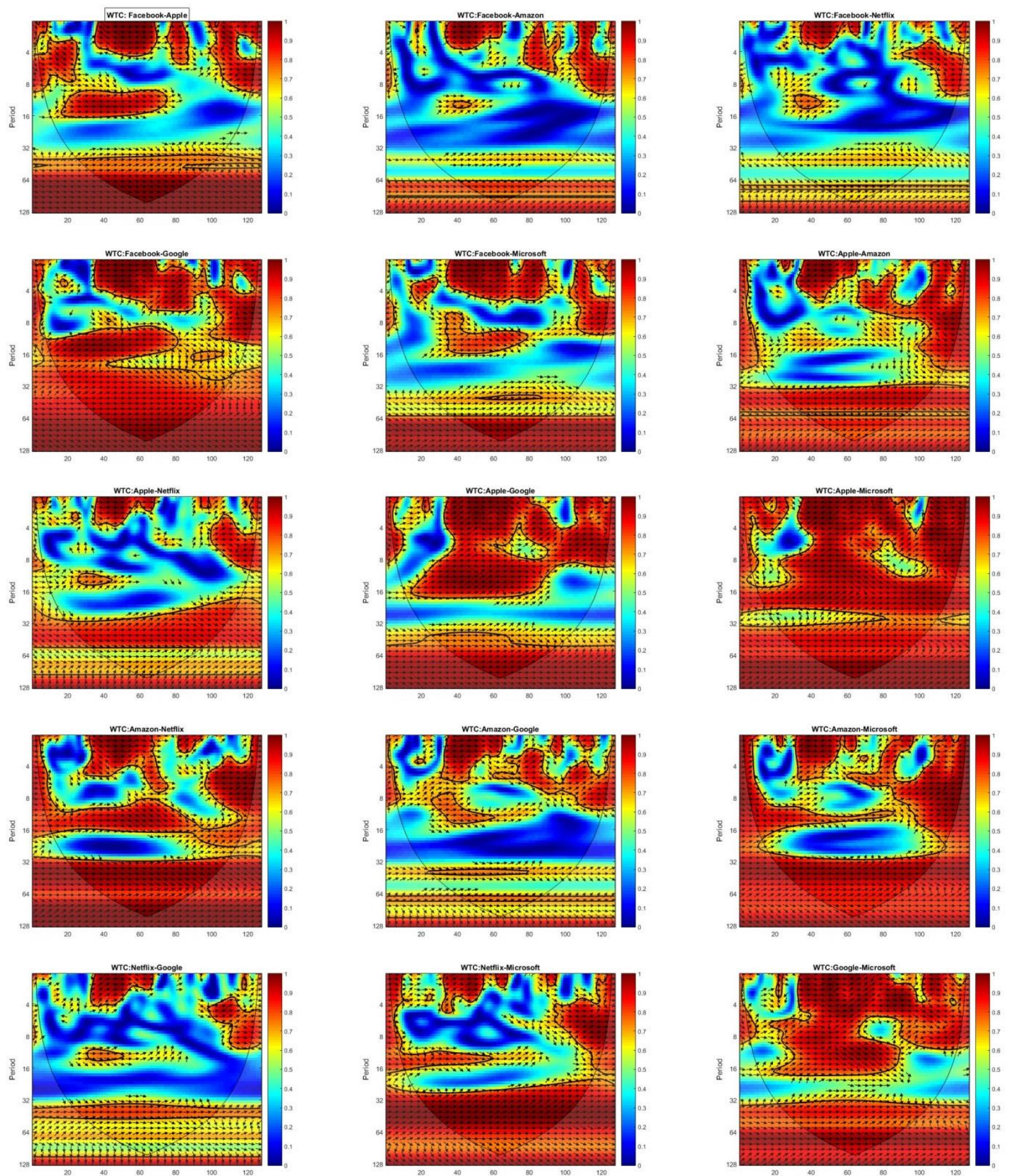

Figure 4: WTC: Facebook-Amazon-Apple-Netflix-Google-Microsoft. Date for COVID-19 period horizontal axis are 1:31.Dec.2019, 20:28.Jan.2020, 40:26.Feb.2020, 60:25.Mar.2020, 80:24.Apr.2020, 100:26.May.2020, 120 


\section{Table 3: FAANG GARCH Models (Overall Period)}

\section{RFACEBOOK} RAPPLE

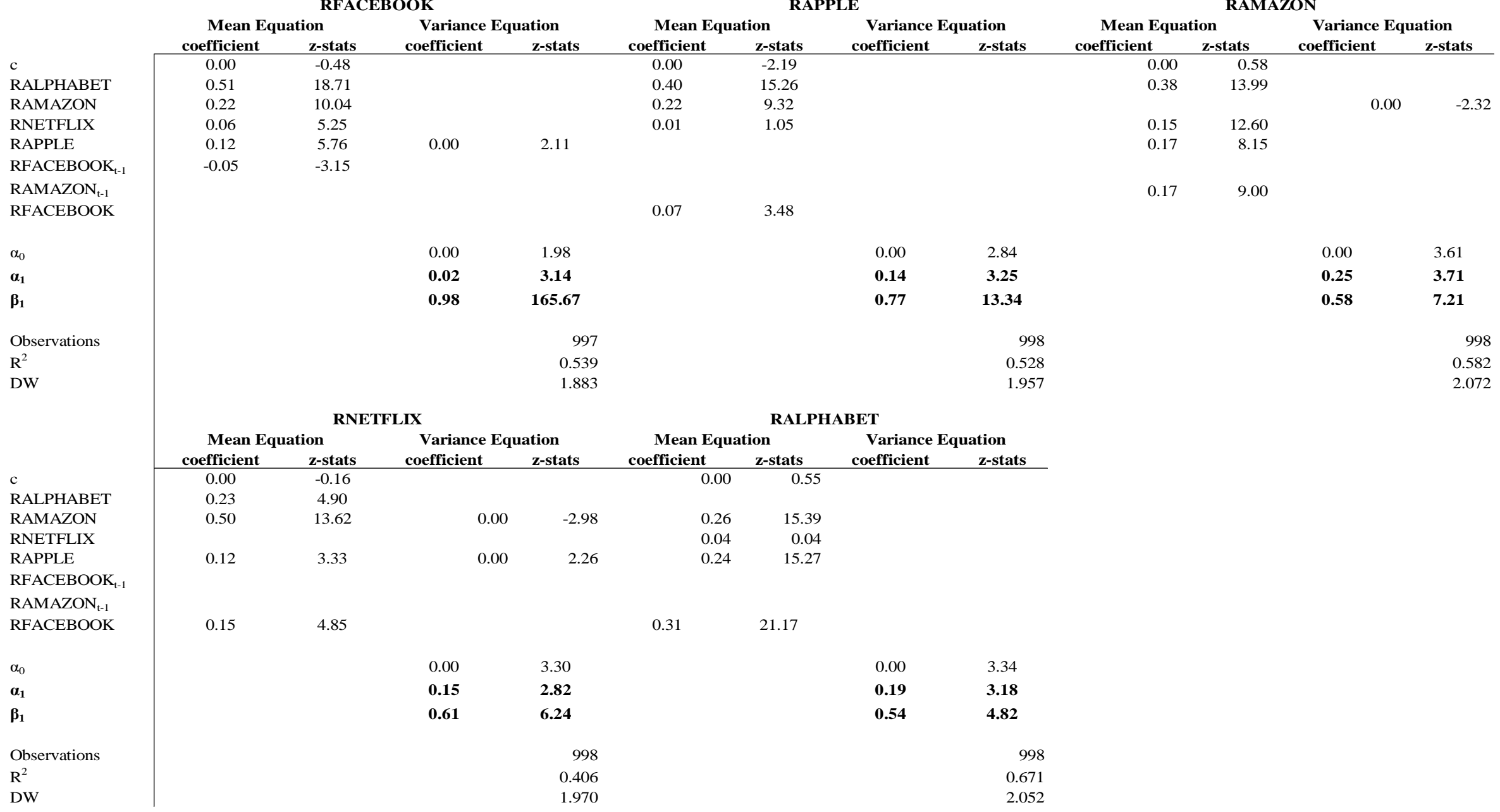


In Table 3 FAANG GARCH models for the overall period are exhibited. Most of the big tech stock returns have a positive impact on the returns of other tech stocks, and they are statistically significant. FAANG models differentiate from each other based on their volatility structure. Only Facebook stock returns are statistically not significant to explain the return changes of Amazon stocks as well as Netflix is statistically not significant to explain the return changes of Alphabet-Google stocks in the overall period. Based on the variance equations of the Facebook model we see that the parameter $\beta$ is 0,9779 and significant at a $1 \%$ level. The sum of $\alpha$ and $\beta$ is 0.99 very close to 1 which shows the persistence of news impact on Facebook stock volatility is very strong. In the Apple variance equation, we see that the parameter $\beta$ is 0,7737 and highly significant. The sum of $\alpha$ and $\beta$ is 0.9100 which shows the persistence of news impact on Apple stock volatility is strong. Moreover, short term persistence is significantly higher for Apple compared to Facebook. For Amazon, Netflix, and Alphabet, the persistence of news impact is not as strong as Facebook and Apple. The sum of $\alpha$ and $\beta$ for Amazon, Netflix, and Alphabet are $0.8304,0.7614$ and 0.7241 , respectively. Furthermore, the value of as of Amazon, Netflix and Alphabet are significantly higher than Facebook and Apple which are $0.2523,0.1529$, and 0.1858 respectfully. These results show that short term shocks have more impact on the volatility of Amazon, Netflix and Alphabet compared to Facebook and Amazon. 
Table 4: FAANG GARCH Models (Covid-19 Period)

RFACEBOOK

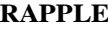

\section{}

Variance Equation Variance Equation
coefficient $\quad$ z-stats z-stats coefficient -stats coefficient Mean Equation RAMAZON

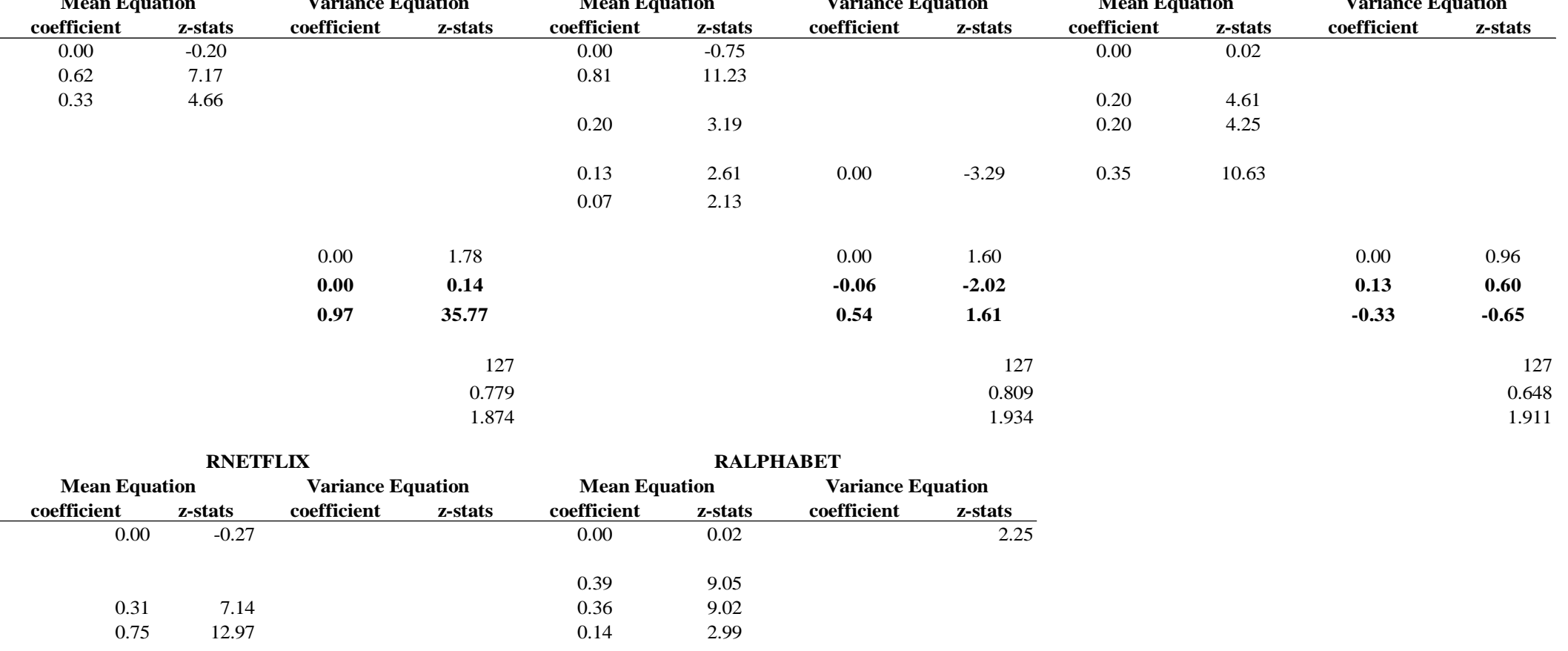

RALPHABET

RAPPLE

RFACEBOOK

RAMAZON

RNETFLIX

RAPPLE $_{(t-1)}$

RALPHABET $_{(\mathrm{t}-\mathrm{l})}$

$\alpha_{0}$

$\alpha_{1}$

Observations

$\mathrm{R}^{2}$

DW

c

RALPHABET

RAPPLE

RFACEBOOK

RAMAZON

RNETFLIX

$\operatorname{RAPPLE}_{(\mathrm{t}-1)}$

RALPHABET $_{(\mathrm{t}-1)}$

$\alpha_{0}$

$\alpha_{1}$

$\beta_{1}$

Observations

$\mathrm{R}^{2}$

DW

$\begin{array}{rrrrr}0.00 & & -0.06 & -1.98 & \\ \mathbf{0 . 7 2} & 2.06 & & 0.00 & 0.78 \\ \mathbf{0 . 0 4} & \mathbf{2 . 7 3} & & \mathbf{0 . 1 6} & \mathbf{1 . 0 1} \\ & \mathbf{0 . 2 1} & & \mathbf{0 . 5 3} & \mathbf{1 . 0 0} \\ & & & \\ & 127 & & \\ & 0.591 & & & 0.869 \\ & 2.229 & & & 2.530\end{array}$


In Table 4 FAANG models for the Covid-19 period are exhibited. We found interesting results. In the pandemic period, the GARCH term became statistically insignificant for all models except Facebook. Also, we detected unit root in the dataset and cointegration relationship among the stocks. Both situations did not exist in the overall period models. In Figure 4, normalized daily stock prices of Facebook, Apple, Amazon, Alphabet, Netflix, and Microsoft are exhibited. In the Covid-19 period, we can see the FAANG stocks and Microsoft stocks behave and move as if they are all one stock. 
Table 5: FAAMG GARCH Models (Overall Period)

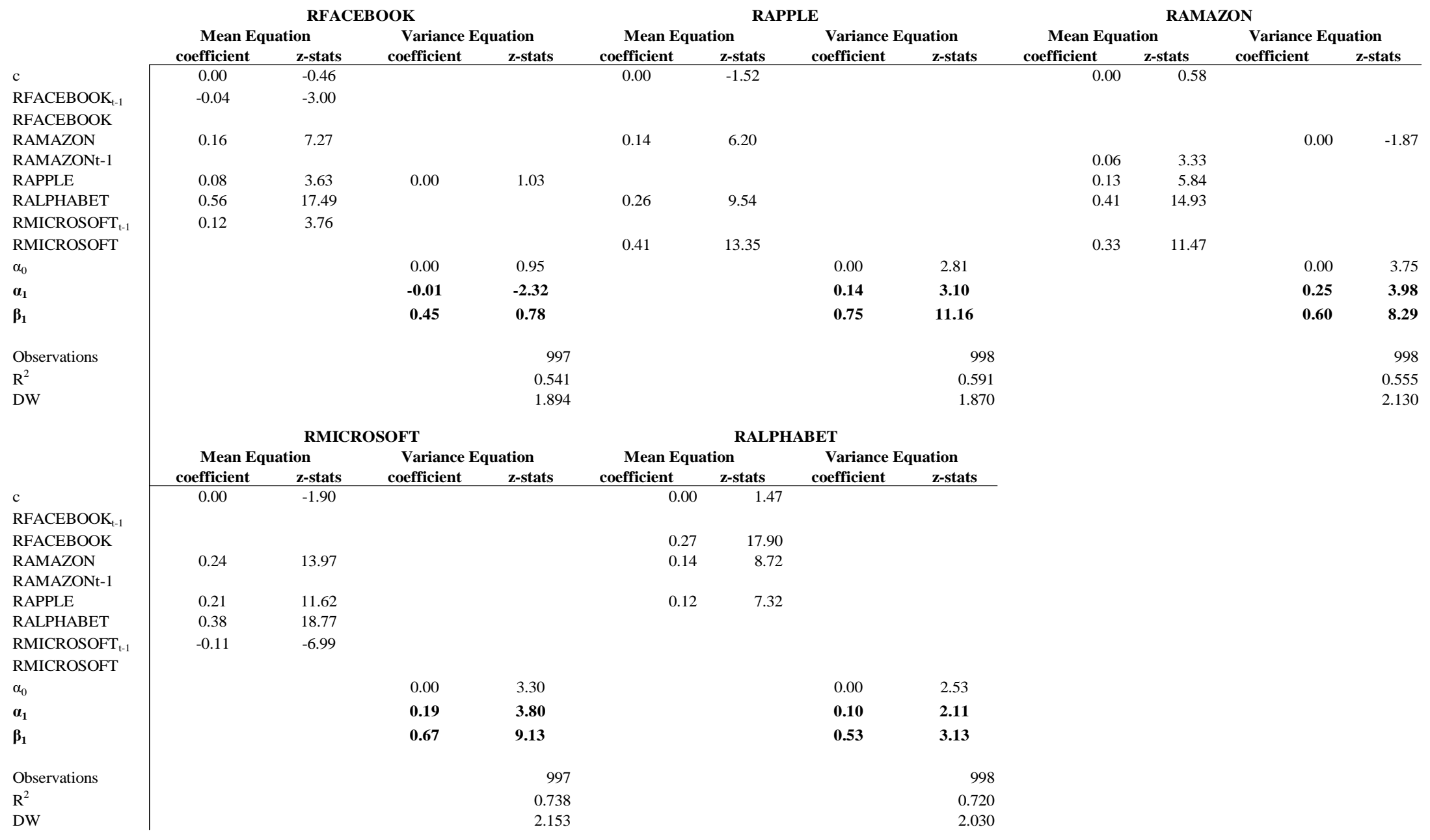


In Table 5 FAAMG GARCH models for the overall period are exhibited. Most of the big tech stock returns have a positive impact on the returns of other tech stocks and they are statistically significant. Only lagged variables of Facebook and Microsoft stock returns are expected to have a negative impact on Facebook and Microsoft returns. FAAMG models differentiate from each other due to their volatility structure. Based on the variance equations of the Facebook model, we see that the parameter $\beta$ is statistically not significant and $\alpha$ is negative. In the Apple variance equation, we see that the parameter $\beta$ is 0,7481 and highly significant. The sum of $\alpha$ and $\beta$ is 0.8900 , which shows the persistence of news impact on Apple stock volatility is strong. Moreover, again short-term persistence is significantly higher for Apple compared to Facebook. For Amazon, Microsoft, and Alphabet, the persistence of news impact is not as strong as Apple. The sum of $\alpha$ and $\beta$ for Amazon, Microsoft, and Alphabet are 0.8494, 0.8578, and 0.6246, respectively. Furthermore, the value of as of Amazon, and Microsoft is significantly higher than Facebook, Apple, and Alphabet which are 0.2466 and 0.1874 respectfully. These results show that short term shocks have more impact on the volatility of Amazon and Microsoft compared to Facebook, Amazon, and Alphabet. In this context, we can conclude that FAANG and FAAMG GARCH models have similar returns and volatility structures which shows that either including Netflix or Microsoft to big tech (FAAG) stocks group does not have a significant difference.

The data that support the findings of this study are available from the corresponding author upon reasonable request. 


\section{References}

[1] Barunik, J., E. and Vacha L. 2013. Contagion among Central and Eastern European stock markets during the financial crisis, 63(5), 443-453

[2] Francq, Christian and Zakoian, Jean-Michel 2010: QML estimation of a class of multivariate GARCH models without moment conditions on the observed process, MPRA Paper No. 20779

[3] Grinsted, A., Moore J. C., and Jevrejeva S. 2004. Application of the cross wavelet transform and wavelet coherence to geophysical time series. Nonlinear Processes in Geophysics 11, 561-566.

[4] Gong, D., Jiang, T., Lu, L., 2020. Pandemic and Bank Lending: Evidence from the 2009 H1N1 Pandemic. Finance Research Letters, 1016127

[5] Goupillaud, P., Grossman, A. and Morlet, J. 1984 Cycle-Octave and Related Transforms in Seismic Signal Analysis. Geoexploration, 23, 85-102.

[6] Mazur, M., Dang, M., Vega, M. 2020 Covid- 19 and the march 2020 stock market crash. Evidence from S\&P1500. Finance Research Letters, forthcoming, 101690

[7] Mirza, N., Naqvi, B., Rahat, B., Rizvi, A, K, S. 2020. Price reaction, volatility timing and funds' performance during Covid-19. Finance Research Letters, forthcoming, 101657

[8] Lau, K.-M., and H.-Y. Weng, 1995: Climate signal detection using wavelet transform: How to make a time series sing. Bull. Amer. Meteor. Soc., 76, 2391-2402.

[9] Torrence, C. and Compo G. P. 1998. A practical guide to wavelet analysis. Bulletin of the American Meteorological Society 79 (1), 61-78.

[10] Tiwari, A. K. 2012, Decomposing Time-Frequency Relationship between Interest Rates and Share Prices in India through Wavelets, MPRA Paper No. 39693

[11] Vacha L., Barunik J. 2012. Co-movement of energy commodities revisited Evidence from wavelet coherence analysis, Energy Economics, Elsevier, 34(1), 241-247. 